\title{
Face Detection and Recognition for Security System using Local Binary Patterns
} (LBP)

\author{
B.A.S.S. Karunarathne ${ }^{1 *}$, W. H. C. Wickramaarachchi ${ }^{2}$, K.K.K.M.C. De Silva ${ }^{3}$ \\ 1, 2, 3 Department of Computing and Information Systems, Wayamba University of Sri Lanka, Kuliyapitiya, Sri Lanka
}

\begin{abstract}
Facial recognition has been used in several areas such as security, detection of criminals or suspects, etc. The face is considered the most important part of the human body in the person identification process since it can be considered the most important perspective in human identification. This paper proposed a real-time monitoring security system for high secured areas rather than relying on human security officers. The proposed system engages with capturing and detecting the human face using the real-time image segment captured by the web camera through the Viola-Jones algorithm. After detecting the face in the captured image, face identification can be made through the LBP by finding the similarities. Once the identification is made, automatically it recognizes the face. Open Computer Vision (OpenCV) is one of the most efficient machine learning libraries used for Object Detection. OpenCV library is used for detection, and Haar - Cascades are trained to detect certain objects. LBP is used for the face recognition process.
\end{abstract}

Index Terms - LBP, Histograms, Feature Extraction, Feature Vector

Received: 23 April 2019; Accepted: 11 May 2019; Published: 26 June 2019

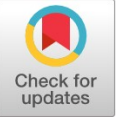

(C) 2019 JITDETS. All rights reserved.

\section{INTRODUCTION}

Today, global security is one of the main functions of society. Nowadays, most of the institutes are facing major security issues when they consider their security. It needs high security for most of the highly secured areas, such as war zones, secured data storage offices, bank lockers, etc. It should have a high-security system which is strictly restricted for the strangers. It will be so easy if we can automatically identify the strangers at first sight. In order to get rid of these problems, they have to assign well-trained security masters. But still, there is a possibility for the human errors which cannot be fixed again. It might be risky for the institutes which need high security [1].

Since human beings make mistakes that might affect the level of security for large institutes, it is better to have a device that can auto-sense, detect and identify its vision.

The proposed solution is to develop a system which can detect strange entry to restricted or high-security areas. The main ideas of the proposed solution are to minimize human errors and labor power. This system consists of two major parts: hardware and software. Hardware part consists of a camera, in order to capture the required information needed for identification, whereas the software part consists of face recognition and face detection algorithms which can process the given information.

Another objective of this research is to open a pathway to the smart world concept. As modern technology stepped into a fully automated world, it is a major concern to implement a security system which automatically operates.

\footnotetext{
${ }^{*}$ Corresponding author: B.A.S.S. Karunarathne

${ }^{\dagger}$ Email: sinalissk@gmail.com
}

\section{LITERATURE REVIEW}

Sandhaya Sharma and Ayush Srivastava have done a research on "Real Time Multiple Face Recognition" using PCA, and calculations are done using Eigen distance. Individual facial outlines in real time are captured by Principle Component Analysis (PCA). Eigen face distance vector is used to detect the face and identify that the identified face is a legitimate face. Then the captured faces are compared with the dataset and then the identification process takes place [2].

Ofor, Nwiabu, and Matthias [3] presented a study on "Face Detection and Expression Recognition Using Fuzzy Rule Interpolation". This research study is aimed at implementing face detection and recognition using fuzzy rule interpolation technique. In this study, emotion recognition is done using the Viola Jones algorithm and LBP algorithm for the feature extraction.

Pentland and Turk [4] introduced face recognition on "Face Recognition Using Eigenfaces". Here they have explained how the eigenface approach is used to simplify and increase the accuracy of person identification. PCA and Eigen faces are used for identification and recognition process.

Elena Alionte and Corneliu Lazar conducted a study on Facial recognition and detection. In the paper, it is stated that the face detection process becomes complex as there are various factors to be considered such as facial expressions, different and illuminations, age variations and facial occlusions [5].

Human beings can see the world and visually sense as well. Several pieces of research have taken place to improve the machines up to human standard. Computer vision is concerned with automatic identification 
and recognition as human beings.

In early 1964 and 1965, Bledsoe, Helen Chan and Charles Bisson started face identification and detection through the computers. Since 1965 , face detection and recognition technology widely expanded with the technology. Even nowadays, Facebook also has provided the facility to automatically identify and tag the persons when you are uploading a photograph. Most of the high-security areas and busy workstations allow developing automated identification in order to reduce human errors $[6,7]$.

The face is the most important part of the body, and that is the easiest way to recognize people. The face is a complex multidimensional structure, so computers need high-quality techniques to recognize a face. There are many more technical aspects improved to detect faces and recognize the person. Face detection and face recognition can be defined as follows:

- Face detection: Here, it performs on detecting a face. It detects from the captured image that which part of the image is a face.

- Face Recognition: Characteristics which describe the image can be found through the face recognition algorithm using the facial image, which is already extracted [8].

There are two main methods where face recognition systems can be performed. Face recognition is one of the most challenging processes in machine learning problems. Some major issues associated with face recognition system are [8]:

I. Illumination problem: Due to the reflexivity of human skin, even a slight change in the illumination of the image can widely affect the re- sults.

II. Pose changes: The performance will be directly affected by any rotation of the head.

III. Time delay: Databases have to be regularly updated as the ageing of human faces.

In order to get rid of those types of problem, three main phases can be identified in face detection. The first step is face representation, then feature extraction and classification. Face representation is,

- Identifying the existence of a face. That is, to determine whether the captured image contains a face.

- The image is transferred to the feature extraction phase. In order to perform further processes, the most useful and unique features of the identified face image are extracted.

- Then it passes to the classification phase carrying the obtained features.

- Then the captured image, which is captured through a real-time system, is compared with the images which are already stored in the database, and threshold values are used to determine if the differences are small enough.

- In order to obtain an accurate output, unique features that are extracted in the feature extraction phase are used in the classification. The output of the classification phase will be identified as a face image or non-identified face image.

Following diagram gives an idea of the face recognition system.

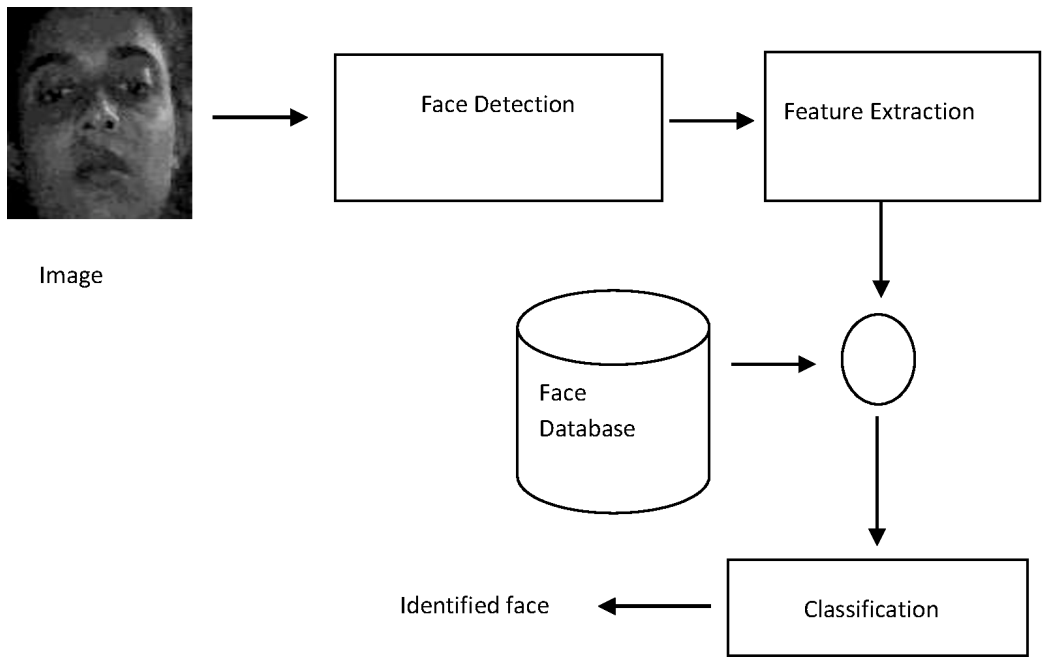

Fig. 1. Conceptual design

\section{A. Face Recognition using LBP}

LBP can be used for classification in computer vision. It has found a strong feature for texture classification method since 1994. LBP was proposed for texture analysis with local features. And also, the shape of a digital image can be easily described with LBP. In order to obtain an accurate result, features should be extracted by dividing the image into several small regions.

The environment of each and every pixel in the image can be described using the features which consist of binary patterns. Histogram can be obtained by concatenating the obtained features, which forms a representation of the image. Images can then be compared by measuring the similarity between their histograms [9].

\section{B. OpenCV}

OpenCV is an open-source computer vision and machine learning software library which was launched in 1999. It consists of many more optimized algorithms [10]. We can make the use of these algorithms to perform detection and recognition of faces, identify the given objects, classify human actions in videos, camera movements tracking, track moving things, extract 3D models of objects, recognize scenery and establish markers to overlay it with augmented reality, etc. OpenCV is written natively in $\mathrm{C}++[11]$. 


\section{Haar-Cascades}

Haar-Cascades are trained to detect a certain type of objects. Haar wavelet is a mathematical fiction that produces square-shaped waves and used to create a box-shaped pattern to recognize signals. Face detection can be performed by combining several Haar-like-features. Several classifiers were combined together to create stronger classifiers.

\section{METHODOLOGY}

Local Binary Pattern Histogram (LBPH) is considered as a texture describer which is useful to symbolize Faces. The face is a multidimensional structure, and it can be split as compositions of patterns of microtextured [12]. Basically, LBPH is carried out in 3 stages:
i. Feature extraction,
ii. Matching,

iii. Classification

\section{A. Face Detection}

In this phase, the main objective is to identify from the given image that which part is the face. This can also be taken as kind of object detection. The face detection is carried out as stages; the first stage is the image capturing and detection using Haar-cascades. Features are checked, and then it is detected as a face. Here, the classifier objects are created using OpenCV [13].

\section{B. Face Recognition Process}

Here, the objective is to identify the person. Using the obtained dataset, the system should be able to identify each person. There are three steps in the recognition process. First, the dataset should be created. Then unique features should be extracted, classifying them and storing in XML files. Finally, matching of the images and prediction should be done.

1) Collecting the image data: Collecting the data for the database is manually done using a camera. Then it starts with a request for a user ID which we can use to store the data in the dataset. A loop runs until the required number of images captured for each person. Then images are automatically stored in greyscale. Then captured images are stored in a dataset folder [10].

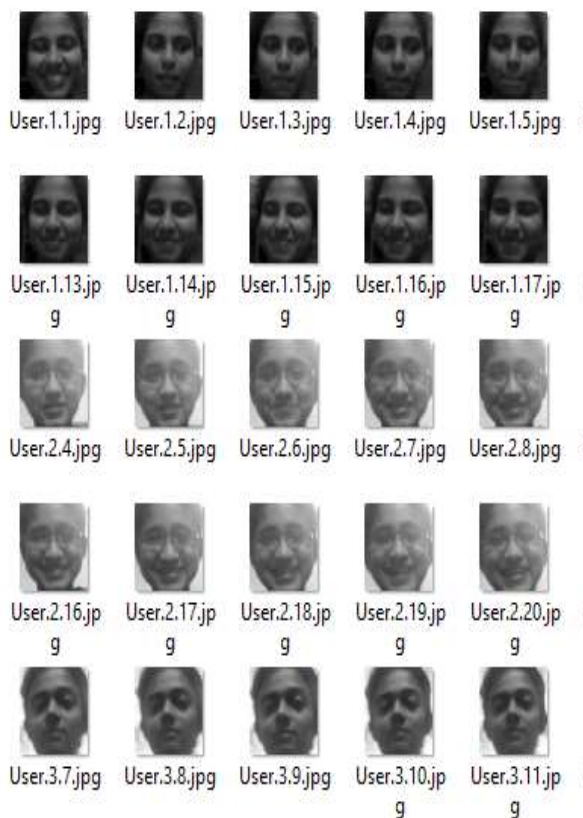

g
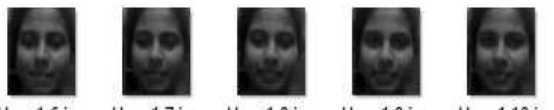

User.1.10
$\mathrm{g}$

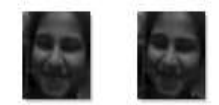

User.1.18.jp
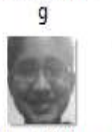

User.2.9.jpg

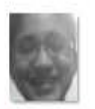

User.2.21.jp

9

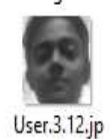

9
User.1.19.jp User.1.20.jp
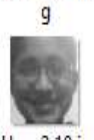

User.2.10.jp

g

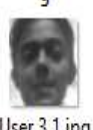

User.3.1.jpg

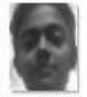

User. 3.13
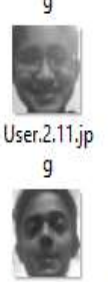

User.3.2.jpg

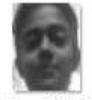

User.3.14.jp

g

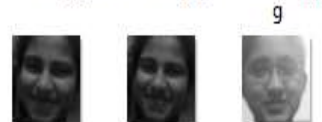

User.1.21.jp User.2.1.jpg

g

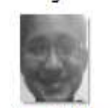

User.2.12.jp

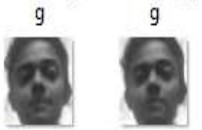

User.3.3.jpg

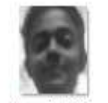

User.3.15.jp

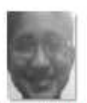

User.2.13.jp

ser.3.4.jpg

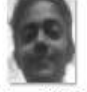

User.3.16.jp

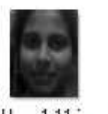

User.1.11.jp User.1.12.jp

g $g$

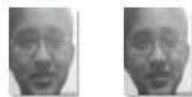

User.2.2.jpg User.2.3.jpg
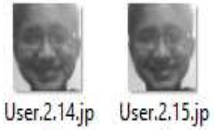

g

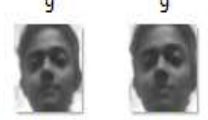

User.3.5.jpg User.3.6.jpg

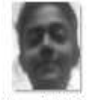

User.3.17.jp User.3.18.jp

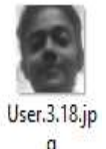

Fig. 2. Snapshot of the dataset

2) Training the classifiers: Classification phase can be conducted to predict the image correctly. This can be taken as one of the major concerns as it is used to identify the person. Using OpenCV, the .xml file is created, and features that are extracted are stored in the folder. Recognizer objects are created, and images are imported and converted into NumPy array and stored in a vector [14].

3) The face recognition: This can be taken as the major phase, as the accuracy of the entire project is depending on the output of this phase.

The face recognition object is created, and the dataset is stored and trained. Then using a web camera, we can capture input images. Once it captured the image, it automatically compares the features with the already extracted features and the best match would be found. Here, Eigen faces, feature extraction and classification take place in order to identify the facial image. Trained classifier is used to identify the person exactly with the trained and extracted features.

Details of each and every authorized person are stored in a database. All the required information is also stored in the database in order to display the details of each and every person when it identified them. Once the trained classifiers object is identified, the names with the IDs are used to display on the screen with the help of already stored data in the database.

The data wanted to be displayed on the screen should be predefined in the database in order to display the list of details on the screen. 


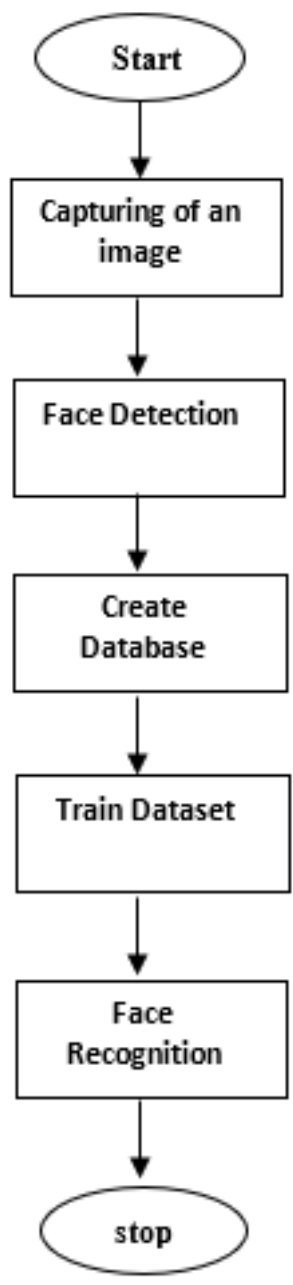

Fig. 3. Flow chat of face detection and recognition process

When a person enters the focused area, a series of snapshots are taken by the existing camera and sent to the software. Then the given data are analyzed and compared with the existing database of already confirmed trusted people. Then the administrator can identify strangers and outsiders automatically. It provides super easy high-security system [15].

\section{RESULTS AND DISCUSSION}

Instead of manual security system, this proposing automated system can be replaced as it contained considerable accuracy. So, the security of the highly secured area can be guaranteed. Then no need to spend more money on training security masters and also no need to face major problems due to human errors.

The experiments have been performed on a manually created training dataset. Dataset can be created through the web camera and then stored in the database. The details of each and every person also can be stored in the database beforehand [16].

Based on the implemented algorithm, the face image of an unknown person is compared with face images of known individuals from a large database, and it produces the output.

When the captured image is not presented in the already stored database, it can be taken as a stranger. Then that person is not allowed to enter the highly secured area. Figure 04 illustrates the input facial images used for correct recognition of the facial area.

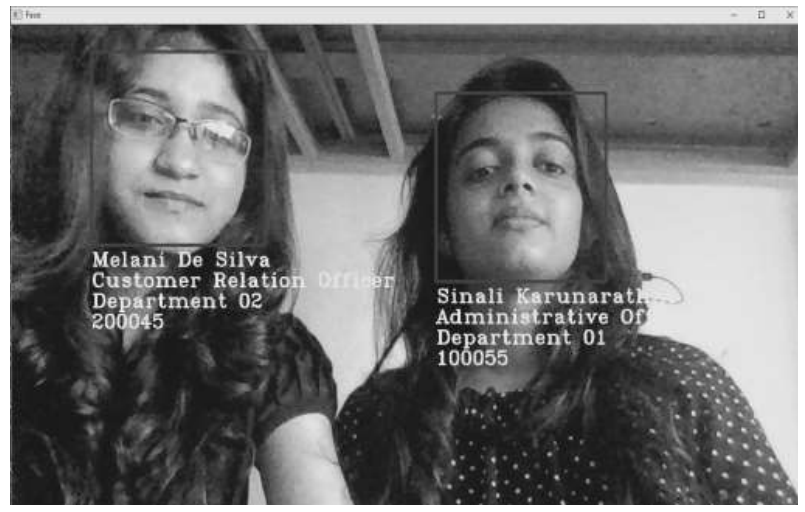

Fig. 4. Correct face identification

In Figure 05, the input facial images used for incorrect recognition both identify as the same person. The background illumination, lighting variations, changing facial expressions, unrecognized poses and quality of the web camera affect the efficiency of the system.

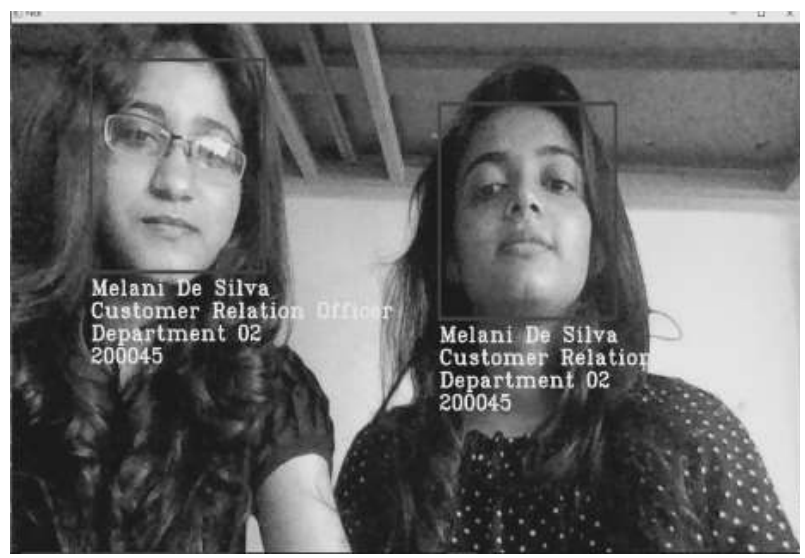

Fig. 5. Incorrect face identification

The system successfully recognizes strangers as well. As in figure 06, it only recognizes those who have saved their information in the database. So, it helps to recognize strangers at first sight.

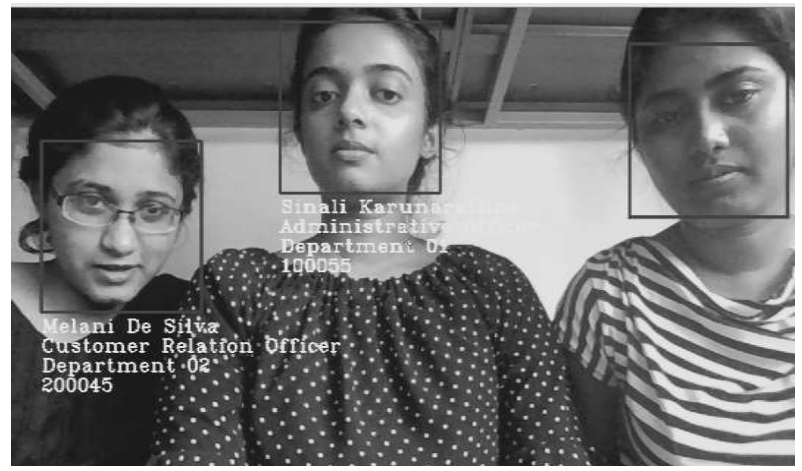

Fig. 6. Correct stranger identification 


\section{CONCLUSION AND IMPLICATIONS}

This research has been done with the use of feature extraction with LBP. It consists of three phases, namely face identification, feature extraction, and classification. After extracting features, this system finally matches the input with the most similar face in the database.

There are many difficulties that we have faced when recognizing face images from the database. Some of them are pose and lighting variations, expression variations, age variations, and facial occlusions. In the future, to improve the quality of the camera, pose correction, quality-based frame selection, and mark-based matching techniques can be combined to build a unified system for face recognition.

\section{Declaration of Competing Interest}

The authors declare that there is no conflict of interest.

\section{References}

[1] W. Zhao, R. Chellappa, P. J. Phillips, and A. Rosenfeld, "Face recognition," ACM Computing Surveys, vol. 35, no. 4, pp. 399-458, 2003. doi: https://doi.org/10.1145/954339.954342

[2] S. Sharma, A. Srivastava, Kaustubh, and K. Singh, "Real time multiple face recognition," International Journal of Advanced Research in Engineering Technology \& Sciences, vol. 2, no. 3, pp. 137-141, 2015.

[3] W. D. Ofor, N. D. Nwiabu, and D. Matthias, "Face detection and expression recognition using fuzzy rule interpolation," International Journal of Computer Sciences and Engineering, vol. 7, no. 5, pp. 1683-1689, 2019. doi: https://doi.org/10.26438/ijcse/v7i5.16831689

[4] M. Turk and A. Pentland, "Eigenfaces for recognition," Journal of Cognitive Neuroscience, vol. 3, no. 1, pp. 71-86, 1991. doi: https://doi. org/10.1162/jocn.1991.3.1.71

[5] M.-H. Yang, D. Kriegman, and N. Ahuja, "Detecting faces in images: A survey," Transactions on Pattern Analysis and Machine Intelligence, vol. 24, no. 1, pp. 34-58, 2002. doi: https://doi.org/10.1109/34. 982883
[6] W. Wójcik, K. Gromaszek, and M. Junisbekov, "Face recognition: Issues, methods and alternative applications," in Face Recognition - Semisupervised Classification, Subspace Projection and Evaluation Methods. London, UK: InTech, 2016.

[7] A. H. Alhussain, "Using deterministic genetic algorithm to provide secured cryptographic pseudorandom number generators," International Journal of Technology and Engineering Studies, vol. 1, no. 4, pp. 107-116, 2015. doi: https://doi.org/10.20469/ijtes.40001-4

[8] D. Meena and R. Sharan, "An approach to face detection and recognition," in International Conference on Recent Advances and Innovations in Engineering (ICRAIE), Jaipur, India, 2016.

[9] M. A. Rahim, M. N. Hossain, T. Wahid, and M. S. Azam, "Face recognition using local binary patterns," Global Journal of Computer Science and Technology, vol. 13, no. 4, pp. 1-8, 2013.

[10] S. Hanamsheth and M. E. Rane, "Face recognition using histogram of oriented gradients," International Journal of Advance Research in Computer Science and Management Studies, vol. 6, no. 1, pp. 29-34, 2018.

[11] P. Y. Kumbhar, M. Attaullah, S. Dhere, and S. Hipparagi, "Real time face detection and tracking using OpenCV," International Journal For Research In Emerging Science And Technology, vol. 4, no. 4, pp. 39-43, 2017.

[12] B. Gupta, A. Chaube, A. Negi, and U. Goel, "Study on object detection using open CV - python," International Journal of Computer Applications, vol. 162, no. 8, pp. 17-21, 2017. doi: https://doi.org/10.5120/ ijca2017913391

[13] OpenCV. About. [Online]. Available: https://bit.ly/3aqu0ya

[14] C. Mounica and P. Venugopal, "face detection and recognition using LBPH," International Journal of Engineereing Research And Science and Technology, vol. 5, no. 3, pp. 1-8, 2016.

[15] S. van der Walt, S. C. Colbert, and G. Varoquaux, "'The NumPy array: A structure for efficient numerical computation," Computing in Science \& Engineering, vol. 13, no. 2, pp. 22-30, 2011. doi: https: //doi.org/10.1109/mcse.2011.37

[16] Python Priinciples. Introduction to python. [Online]. Available: https://bit.ly/39r5nA6 